\title{
Robot-assisted intravertebral augmentation corrects local kyphosis more effectively than a conventional fluoroscopy-guided technique
}

\author{
Sultan Alsalmi, MD, Cyrille Capel, MD, PhD, Louis Chenin, MD, Johann Peltier, MD, PhD, and \\ Michel Lefranc, MD, PhD
}

Department of Neurosurgery, Amiens Picardie University Medical Center, Amiens, Hauts de France, France

\begin{abstract}
OBJECTIVE Intravertebral augmentation (IVA) is a reliable minimally invasive technique for treating Magerl type A vertebral body fractures. However, poor correction of kyphotic angulation, the risk of cement leakage, and significant exposure to radiation (for the surgeon, the operating room staff, and the patient) remain significant issues. The authors conducted a study to assess the value of robot-assisted IVA (RA-IVA) for thoracolumbar vertebral body fractures.
\end{abstract}

METHODS The authors performed a retrospective, single-center study of patients who had undergone RA-IVA or conventional fluoroscopy-guided IVA (F-IVA) for thoracolumbar vertebral body fractures. Installation and operating times, guidance accuracy, residual local kyphosis, degree of restoration of vertebral body height, incidence of cement leakage, rate of morbidity, length of hospital stay, and radiation-related data were recorded.

RESULTS Data obtained in 30 patients who underwent RA-IVA were compared with those obtained in 30 patients who underwent F-IVA during the same period (the surgical indications were identical, but the surgeons were different). The mean $\pm S D$ installation time in the RA-IVA group $(24 \pm 7.5$ minutes $)$ was significantly shorter $(p=0.005)$ than that in the F-IVA group ( $26 \pm 8$ minutes). The mean operating time for the RA-IVA group ( $52 \pm 11$ minutes) was significantly longer $(p=0.026)$ than that for the F-IVA group $(30 \pm 11$ minutes). All RA-IVAs and F-IVAs were Ravi's scale grade A (no pedicle breach). The mean degree of residual local kyphosis $\left(4.7^{\circ} \pm 3.15^{\circ}\right)$ and the percentage of vertebral body height restoration $(63.6 \% \pm 21.4 \%)$ were significantly better after RA-IVA than after F-IVA $\left(8.4^{\circ} \pm 5.4^{\circ}\right.$ and $30 \% \pm 34 \%$, respectively). The incidence of cement leakage was significantly lower in the RA-IVA group $(p<0.05)$. The mean length of hospital stay after surgery was 3.2 days for both groups. No surgery-related complications occurred in either group. With RA-IVA, the mean radiation exposure was $438 \pm 147 \mathrm{mGy} \times \mathrm{cm}$ for the patient and $30 \pm 17 \mathrm{mGy}$ for the surgeon.

CONCLUSIONS RA-IVA provided better vertebral body fracture correction than the conventional F-IVA. However, RAIVA requires more time than F-IVA.

https://thejns.org/doi/abs/10.3171/2018.8.SPINE18197

KEYWORDS robot-assisted; vertebral body fracture; spine; fluoroscopy; intravertebral augmentation; surgical technique

I NTRAVERTEBRAL augmentation (IVA) is a reliable minimally invasive technique for treating Magerl type A vertebral body fractures. ${ }^{8}$ However, poor correction of kyphotic angulation, ${ }^{5}$ the risk of cement leakage, ${ }^{2}$ and significant exposure to radiation (for the surgeon, the operating room staff, and the patient) remain significant issues when using a conventional, 2D fluoroscopy-guided technique. ${ }^{16}$ IVA provides fast pain control and corrects the kyphotic angulation resulting from the fracture; this is important for maintaining normal sagittal balance, which has been shown to be one of the main causes of back pain in long-term follow-up studies. ${ }^{3}$ However, vertebral body height can be hard to restore with this technique. ${ }^{9}$ Furthermore, cement leakage is associated with other complications. ${ }^{18}$ Additionally, fluoroscopy-guided techniques expose the surgeon, the operating room staff, and the patient

ABBREVIATIONS F-IVA = fluoroscopy-guided intravertebral augmentation; $\mathrm{fpCT}=$ flat-panel $\mathrm{CT} ; \mathrm{IVA}=$ intravertebral augmentation; LOS $=$ length of hospital stay; $\mathrm{NS}=$ not significant; PMMA = polymethylmethacrylate; RA-IVA = robot-assisted IVA; VAS = visual analog scale.

SUBMITTED February 18, 2018. ACCEPTED August 1, 2018.

INCLUDE WHEN CITING Published online November 30, 2018; DOI: 10.3171/2018.8.SPINE18197. 
to significant doses of ionizing radiation exposure; cancer rates are abnormally high among neurosurgeons, spine surgeons, and surgically treated patients. ${ }^{22}$

Navigation with cone-beam CT systems, such as the Oarm navigation system, and robotic devices is increasingly being used to decrease the level of radiation exposure, ${ }^{10}$ improve safety, and increase the accuracy of guidance and thereby decrease the degree of cement leakage. ${ }^{12}$

However, there are no published comparative data on fluoroscopy-guided versus navigated IVA techniques, and the potential value of robotic assistance for increasing the reliability and quality of vertebral body height restoration has not previously been assessed.

Robotic assistance allows for 3D planning of the Kwire's position, and it might thus improve the implant's placement within the vertebral body. Additionally, robotic assistance might (like other navigational techniques) minimize the extent to which the surgical team and the patient are exposed to ionizing radiation.

The objective of the present study was to assess the value of robot-assisted IVA (RA-IVA) for thoracolumbar body fracture.

\section{Methods}

We performed a descriptive, retrospective, comparative, single-center study of patients who underwent vertebral body augmentation for the treatment of Magerl type A3.1/ A3.2 or type A2.1/A2.2/A2.3 traumatic vertebral fractures $^{8}$ with either RA-IVA or conventional 2-dimensional fluoroscopy-guided IVA (F-IVA). Only fractures treated with a SPINEJACK device (Vemix $)^{17}$ were included in the study to avoid any variability in body height restoration associated with the choice of implant. The size of the implant was based on pedicle size (a small implant " 4.2 " for a pedicle size smaller than $5 \mathrm{~mm}$ in diameter, a medium " 5 " implant for those $5-6.5 \mathrm{~mm}$ in diameter, and a large implant " 5.8 " for those greater than $6.5 \mathrm{~mm}$ in diameter).

Data obtained in 30 patients treated with RA-IVA were compared with those obtained in 30 patients treated with F-IVA during the same period between January 2015 and January 2016 at Amiens Picardie University Medical Center (Amiens, France). All patients were examined 6 weeks after surgery and then at 6-9 months after surgery.

The primary endpoint of the study was the postoperative correction of the vertebral body height. The degree of residual local kyphosis was defined as the angle between the upper endplate of the vertebral body and the lower endplate of the vertebral body of the fractured vertebra. The radiological measurements were done most commonly by two independent spine surgeons. Figure 1 illustrates the measurements before and after surgery.

The percentage of vertebral body height restoration was calculated using the following formula:

$$
[(\mathrm{a}-\mathrm{b}) / \mathrm{a}] \times 100 \text {, }
$$

where $a$ is the local kyphosis angle before IVA and $b$ is the local kyphosis after IVA. Angle a was calculated from preoperative CT scan, and angle $\mathrm{b}$ was calculated from a 3D CT scan acquired at the end of the surgical session for the RA-IVA group and from a CT scan obtained 1 or 2 days after surgery for the F-IVA group.
After surgery, the accuracy of K-wire insertion was rated on the Ravi scale ${ }^{19}$ (A, no pedicle breach; B, pedicle wall breach of less than $2 \mathrm{~mm}$; $\mathrm{C}$, pedicle wall breach of 2-4 $\mathrm{mm}$; and $\mathrm{D}$, pedicle wall breach of greater than $4 \mathrm{~mm})$.

The incidence of cement leakage was noted and classified into 1 of 4 grades: A, no cement leakage; B, minimal cement leakage; $\mathrm{C}$, marked cement leakage with no clinical consequences; and D, marked cement leakage, with clinical consequences. ${ }^{2}$

Radiation exposure during RA-IVA and F-IVA was also analyzed. We were not able to obtain intraoperative fluoroscopic radiation data for the F-IVA group and therefore only compared doses from 3D imaging.

Each procedure was performed by one of two experienced consultant neurosurgeons between January 2015 and January 2016. After the procedure, 3D scans were acquired to assess the local residual kyphosis and cement leakage. The length of hospital stay (LOS), the installation time, the operating time, and the morbidity rate were also recorded.

\section{Operating Technique: Conventional 2D F-IVA}

Patients were placed in the prone position after the induction of general anesthesia. The damaged vertebra and the needle position were localized on anteroposterior and lateral fluoroscopic images. After insertion of the K-wire with a Jamshidi needle (percutaneous transpedicular approach with fluoroscopic guidance), the vertebra was cannulated using a SPINEJACK device that was gradually expanded (like a jack) using a specially designed tool (part of the expansion kit). The tool locks into the device and pulls the axial ends of the implant toward each other. Turning the tool clockwise causes the implant to open in the inferosuperior direction only (due to its machined grooves). Expansion of the device applies a 500-N distraction force to the fracture, along the craniocaudal axis, similar to a jack. Device expansion is achieved using a tool that pulls the two ends of the implant toward each other, shortening the device and deploying the central titanium component. A rack-and-pinion system blocks the expansion of the implant at the desired height while preventing any loss of correction before the injection of polymethylmethacrylate (PMMA), which envelops the implant, ensuring definitive stabilization of the fracture. A simple mechanism locks the implant into the desired expanded position, as determined and controlled by the surgeon. Once the desired degree of expansion has been achieved, the device is left in place inside the restored vertebra, and PMMA bone cement is injected into and around the implant.

Anteroposterior and lateral fluoroscopic images were used to check the position throughout the implant insertion/expansion and cement injection. However, 3D scans were acquired 1 or 2 days after surgery.

\section{Operating Technique: RA-IVA}

We performed IVA with a robot-assisted, minimally invasive technique. The ROSA robot (Medtech) was combined with intraoperative flat-panel CT (fpCT) guidance (O-arm, Medtronic). Patients were placed in the prone position on a radiotransparent spinal operating table after the 

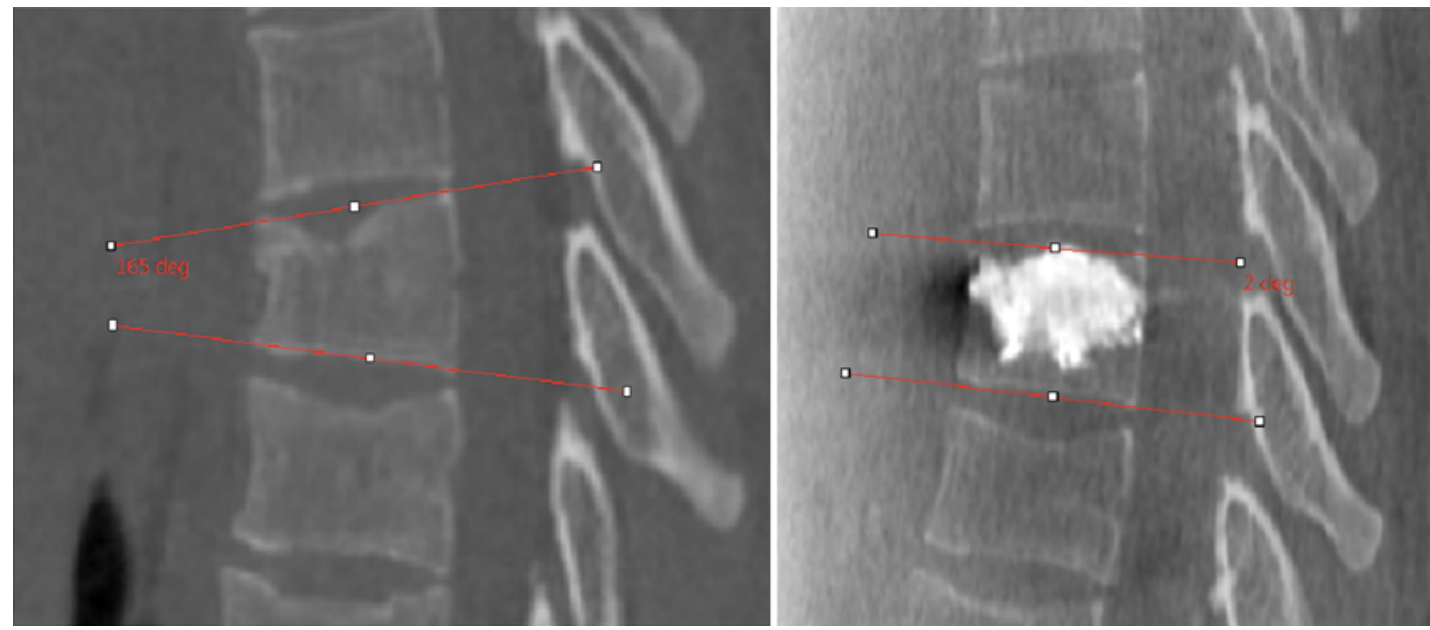

FIG. 1. Sagittal CT scans obtained before and after surgery. Figure is available in color online only.

induction of general anesthesia. The O-arm CT scanner and the ROSA Spine robot were put in place. Lateral and anteroposterior fluoroscopic images were used to localize the target vertebra. As with all minimally invasive neuronavigation procedures, a percutaneous reference frame with reflective markers for navigation was attached to the posterior iliac crest through a paramidline incision (for a lumbar fracture) or to the spinous process through a small midline incision (for a thoracic fracture). 3D images from the O-arm were transferred to the ROSA Spine's adjunct surgical workstation. First, the 3D trajectory for bilateral transpedicular K-wire placement was planned. By controlling the positioning of the K-wire and the implant, we can estimate our ability to reduce the fracture (based on the intraoperative imaging assessments). Using the planning software, we chose the implant's position for optimal reduction of the vertebral body fracture (anteroposterior placement, height in the vertebral body, and angulation with respect to the broken endplate). The skin incision site was determined with assistance from the robotic arm, including the extension of the desired transpedicular trajectory. Second, a cannulated reducer held by the robot was placed in front of the pedicle entry point along the planned trajectory, and a hole through the cannulated reducer was drilled through each pedicle using real-time robotic guidance; the robot is able to track the movements of the patient's body in real time. The K-wire through the cannulated reducer was passed through the skin and the pedicle and was then tapped into the anterior part of the vertebral body. The guidewire tapping step is navigated and monitored by the robot in real time. The robot tracks the instruments' exact spatial positions throughout the surgical session. A second hole was drilled through the second pedicle, and a second guidewire needle was inserted using the same technique (again using real-time, computeraided, robot-assisted navigation). Once the guidewire had been put in place, the rest of the IVA procedure was performed in a standard fashion (as in the F-IVA). Implant insertion/expansion and PMMA cement injection were guided by the fluoroscopy mode of the fpCT. After the cement had been injected, a final intraoperative 3D fpCT scan was acquired in order to evaluate the vertebral height, and any leakage of cement into the disc space, paravertebral soft tissue, vascular system, or spinal canal. A video of the robot-assisted technique is shown in Video 1.

VIDEO 1. Video showing the RA-IVA technique, coupling robotic assistance and intraoperative cone-beam CT scanning. Copyright $\mathrm{CHU}$ Amiens Picardie University Hospital. Published with permission. Click here to view.

Figures 2 and 3 illustrate the RA-IVA technique.

\section{Statistical Analysis}

A bivariate, nonparametric Wilcoxon test was used to compare independent, quantitative variables (with a minimum sample size of 8 per group). Fischer's exact test was used to compare independent, qualitative variables (for small sample sizes). The threshold for statistical significance was set to $\mathrm{p}<0.05$. Mean values are presented \pm SD .

\section{Results \\ Descriptive Data}

The RA-IVA group included 16 men and 14 women, with a mean age of 49.4 years (range 17-68 years). The surgery involved 7 thoracic vertebrae and 28 lumbar vertebrae. The F-IVA group included 11 men and 19 women, with a mean age of 40.6 years (range 18-66 years). The surgery involved 10 thoracic vertebrae and 22 lumbar vertebrae. The two groups were similar in terms of the vertebrae treated and the degree of local kyphosis before surgery (Table 1). The mean preoperative visual analog scale (VAS) pain score was $6.8 \pm 1.3$ in the F-IVA group. The mean preoperative VAS pain score was $6.9 \pm 12$ in the RA-IVA group. The scores were not significantly different between the groups $(\mathrm{p}=0.66)$.

\section{Installation and Operating Times}

The mean installation time was $24 \pm 7.5$ minutes in the RA-IVA group and $26 \pm 8$ minutes in the F-IVA group (p $=$ not significant $[\mathrm{NS}])$. The mean operating time was 52 

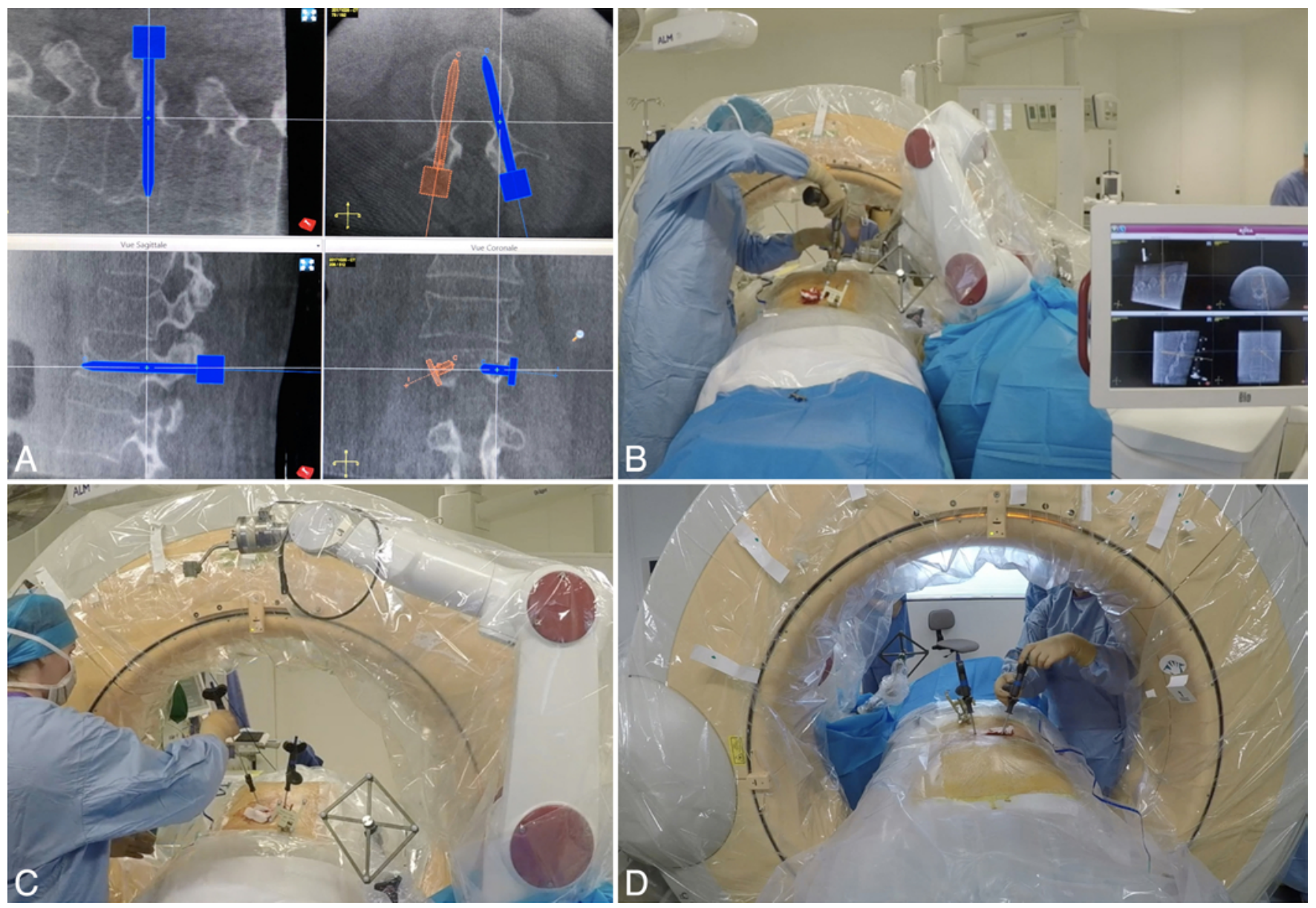

FIG. 2. Illustration of the RA-IVA technique. A: The 3D trajectory for bilateral transpedicular K-wire placement is planned. B: A hole was drilled through each pedicle using real-time robotic guidance; the robot is able to track the movements of the patient's body in real time. The K-wire was passed through the skin and the pedicle, and was then tapped into the anterior part of the vertebral body. C and D: Implant insertion/expansion and PMMA cement injection were guided by the fluoroscopy mode of the fpCT scanner. Figure is available in color online only.

\pm 11 minutes in the RA-IVA group and $30 \pm 11$ minutes in the F-IVA group ( $\mathrm{p}<0.005)$. In the RA-IVA group, we observed that the operating time declined over the study period; it was significantly lower for the last 8 operations than for the first 8 (mean 44 vs 56 minutes, respectively; $\mathrm{p}=0.026$ ).

\section{Accuracy of Guidance, Degree of Local Residual Kyphosis, Percentage of Restoration of Vertebral Body Height, and Incidence of Cement Leakage}

All RA-IVA procedures and all F-IVA procedures were Ravi's scale grade A (no pedicle breach). In the RAIVA group, the mean local residual kyphosis after surgery was $4.7^{\circ} \pm 3.15^{\circ}$ and percentage of restoration of vertebral body height was $63.6 \% \pm 21.4 \%$. In the F-IVA group, the mean degree of local residual kyphosis after surgery was $8.4^{\circ} \pm 5.4^{\circ}$, and the percentage of restoration of vertebral body height was $30 \% \pm 34 \%$; this was significantly lower than that in the RA-IVA group ( $p<0.005)$. In the subgroup of patients who underwent thoracic-level treatment, the percentage of vertebral body height restoration was still significantly better in the RA-IVA group $(\mathrm{p}=0.05)$.

\section{Clinical Outcome, Morbidity, and LOS}

All patients had a favorable course immediately after surgery, and follow-up consultations at 6 weeks and then at 6-9 months after surgery confirmed the quality of the outcomes; there was no mortality or major morbidity (e.g., infections, embolism, or neurological impairment) in either group. No device- or surgery-related complications were observed. The mean postoperative VAS scores were $2.56 \pm 1.0$ in the RA-IVA group and $2.55 \pm 1.1$ in the FIVA group. The level of pain was significantly lower after surgery for both groups $(\mathrm{p}<0.0001$ for RA-IVA and $\mathrm{p}<$ 0.0001 for F-IVA), but it was not significantly different ( $\mathrm{p}$ $=0.9$ ) between the groups. In both the RA-IVA and F-IVA groups, the mean LOS after surgery was 3.2 days.

\section{Incidence of Cement Leakage}

The incidence of cement leakage was significantly lower in the RA-IVA group (45.8\%) than in the F-IVA group $(71.9 \%)(\mathrm{p}<0.05)$. In the RA-IVA group, all cases of cement leakage were grade $\mathrm{B}$, whereas in the F-IVA group, there were 20 grade $\mathrm{B}$ cases and 3 grade $\mathrm{C}$ cases. 


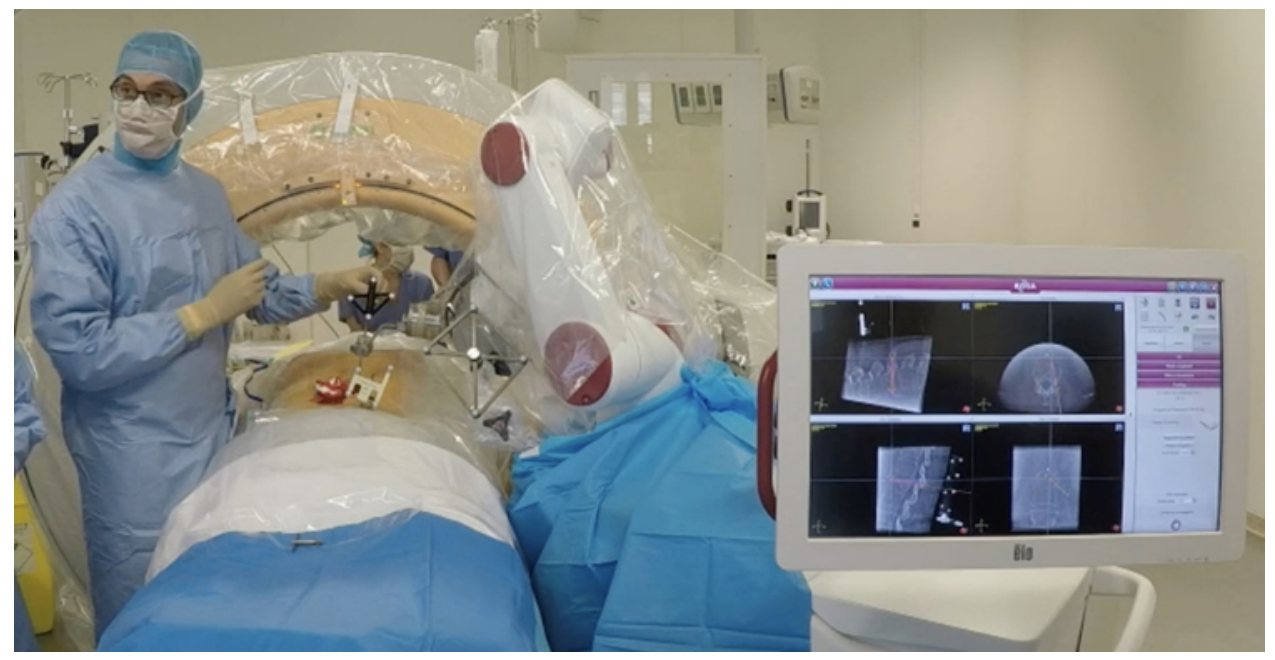

FIG. 3. Illustration of K-wire insertion thanks to robotic assistance. The robot is placed along the planned trajectory. A reducer is placed along the trajectory with regard to the pedicle entry point. The K-wire is passed through the reducer through the skin and pedicle and is navigated into the anterior part of the vertebral body. Figure is available in color online only.

\section{Radiation Exposure}

In the RA-IVA group, the mean radiation exposure was $438 \pm 147 \mathrm{mGy} \times \mathrm{cm}$ for the patient (including 2 intraoperative $\mathrm{fpCT}$ scans-one for the planning and registration process and one for final 3D control) and $30 \pm 17 \mathrm{mGy}$ for the surgeon. In the F-IVA group, we were not able to establish the fluoroscopy dose; the mean dose (3D postoperative CT scan) was $566 \pm 112 \mathrm{mGy} \times \mathrm{cm}$ per patient and was significantly higher than that in the RA-IVA group ( $p$ $=0.0046)$.

\section{Discussion}

Intravertebral augmentation is a reliable minimally invasive technique for treating Magerl type A vertebral body fractures. ${ }^{8}$ However, this technique has several important drawbacks, such as exposure to ionizing radiation, poor restoration of vertebral body height, and cement leakage. ${ }^{23}$ The objective of the present study was to determine whether or not robotic assistance could help to reduce these issues safely and reliably.

Sagittal balance and the correction of posttraumatic kyphosis appear to be important factors in a patient's overall improvement after thoracolumbar spine fractures. Correction of the kyphotic angle is a key parameter linked to back pain during follow-up and to the resumption of professional activities. This may be particularly problematic for a young patient with a vertebral compression fracture. ${ }^{6,15}$ Most research has focused on the relationship between the type of implant and the correction of kyphotic deformation. ${ }^{11}$ Here, we showed that robotic assistance can significantly increase kyphotic correction, relative to a conventional fluoroscopy-guided technique. We did not observe a difference in clinical outcomes between the two methods; however, we can consider that this lack of a difference was due to our study's short follow-up period (1 year).

The advent of neuronavigation and robotics techniques has led to further progress in spine surgery, notably in terms of reducing the morbidity specifically associated with poor screw positioning. ${ }^{3,7,14}$ When compared with conventional fluoroscopy-guided techniques, neuronavigation provides a higher rate of accurate pedicle screw placement (in $68.1 \%$ vs $95 \%$ of cases, respectively). $3,7,14$ Here, we report on the use of a new robotic device (ROSA robot) to

TABLE 1. Summary of the main findings between F-IVA and RA-IVA

\begin{tabular}{|c|c|c|c|}
\hline Variable & RA-IVA & F-IVA & $p$ Value* \\
\hline Mean age (range), yrs & $49.4(17-68)$ & $40.6(18-66)$ & 0.03 \\
\hline Female/male ratio & $14: 16$ & 19:11 & 0.3 \\
\hline \multicolumn{4}{|l|}{ VAS score } \\
\hline Preop & 6.9 & 6.8 & 0.66 \\
\hline Postop & 2.56 & 2.55 & 0.9 \\
\hline No. of levels & 1.16 & 1.06 & 0.8 \\
\hline Thoracic vertebrae & 7 & 10 & 0.4 \\
\hline Lumbar vertebrae & 28 & 22 & 0.4 \\
\hline \multicolumn{4}{|l|}{ Degree of kyphosis } \\
\hline \multicolumn{4}{|l|}{ Preop } \\
\hline All & $12.9^{\circ}$ & $12^{\circ}$ & 0.49 \\
\hline Thoracic subgroup & $16^{\circ}$ & $13.1^{\circ}$ & 0.18 \\
\hline Postop & $4.4^{\circ}$ & $8.4^{\circ}$ & 0.0049 \\
\hline Thoracic subgroup & $5.9^{\circ}$ & $9.3^{\circ}$ & 0.05 \\
\hline $\begin{array}{l}\text { Mean local kyphosis cor- } \\
\text { rection }\end{array}$ & $63.6 \%$ & $30.0 \%$ & 0.0049 \\
\hline Thoracic subgroup & $57.4 \%$ & $30.6 \%$ & 0.05 \\
\hline Cement leaks & $45.8 \%$ & $71.9 \%$ & 0.0046 \\
\hline Thoracic level & $50 \%$ & $70 \%$ & 0.7 \\
\hline Morbidity \& mortality & $0 \%$ & $0 \%$ & NA \\
\hline Days discharged postop & 3.2 & 3.3 & 0.8 \\
\hline
\end{tabular}

NA = not applicable.

${ }^{*}$ Statistical significance was set at $p<0.05$. 
place expandable vertebral body implants. First, the robot tracks and adjusts for all the patient's movements (notably respiratory motion). This is of significant value because the spine is a dynamic system that can move slightly during surgery. The robot also monitors movements induced by the surgeon. It is particularly useful to be able to save all the trajectories in the robot's memory, place the K-wire, and move from one trajectory to another without the need for fluoroscopic guidance. Second, the device used in the present study is a development of the ROSA Brain robot used in brain surgery. The ROSA Spine robot is designed to have the same level of precision in spinal surgery as in stereotactic brain surgery. ${ }^{12,13}$ Trajectories are planned in the same way as in the brain system, with specification of the implant's entry point, direction, and final position. This feature enables the surgeon to optimize implant placement by, for example, optimizing the implant position inside the vertebra in 3D visualization, thus reducing the fractured fragments as much as possible. The end of the operation is the same as in conventional minimally invasive fluoroscopic implantation, when fluoroscopy is used to assess the correct expansion of the implant and the spreading of PMMA cement within the vertebra. The greatest advantage of using a robot is its ability to place the implant exactly as planned by the surgeon. As in the neuronavigation system, percutaneous screw placement by the robot enables the implant's ancillary progression into the vertebra to be visualized. To this end, the guidewire is also navigated, and its position within the vertebra is regularly checked (to avoid piercing the anterior wall). The robot clearly has value for optimal implant positioning. The present cases represented perfectly positioned implants. Pedicle breach was never observed on the intraoperative CT scan. Furthermore, the reduction of the fracture was excellent (and better than the one obtained with F-IVA). Nevertheless, some aspects of the RA-IVA procedure could be improved. The graphic interface used to visualize the implant should be improved, with, for example, a virtual view of the implant inside the vertebral body.

We observed a marked difference in cement leakage rates between the RA-IVA group and the F-IVA group. Even though cement leakage was not correlated with an elevated morbidity rate in the present study, specific morbidity has been reported in the literature. ${ }^{21}$ One limit of our study is that we could not calculate the amount of cement injected in each patient. We usually put 4 injectors ( 8 $\mathrm{ml}$ ), and we stop the injection before the entire volume has been used, when we see evident cement leakage on fluoroscopy. As a result, we do not know if the same volume of cement was injected in each group. However, leakage during a surgical procedure prompts cessation of the injection, and so the leaked cement does not contribute to the biomechanical restoration of vertebral body strength.

A 2014 study of computer-navigated kyphoplasty focused on reducing the extent to which the surgeon, patient, and surgical staff were exposed to radiation. ${ }^{22}$ In terms of radiation issues, our robotic device coupled with intraoperative cone-beam CT is very similar to the cone-beam CT navigation technique. In the current study, we found that the radiation exposure for RA-IVA was in line with reports in the literature and was significantly lower than it was for F-IVA. ${ }^{1}$ When we take into account that patients undergoing F-IVA required for their follow-up a postoperative CT scan, which is our routine, the associated dose was significantly greater in F-IVA group. It must be kept in mind that patients undergoing F-IVA require a followup standard CT scan after surgery; the associated dose is greater than for 2 intraoperative fpCT scans acquired during the RA-IVA procedure ${ }^{4,20}$ As described by Barzilay et al., robotic assistance in IVA surgery appears also to be a "radiation reduction tool."1

Although the operating time was longer for RA-IVA than for F-IVA, we consider this a small price to pay for the above-mentioned advantages. Given that RA-IVA is a new technique with a learning curve, we were pleased to observe a significant decrease in operating time for the last procedures to be performed.

Finally, this new RA-IVA technique is as safe as F-IVA. It has the same follow-up in the postoperative period. A lumbar radiograph under weight-bearing conditions is obtained at 3 and 6 months after surgery. Lumbar CT scanning and EOS imaging are conducted at 1 year to evaluate sagittal balance of the spine and local kyphosis. The present observational study generated promising short- and midterm outcomes, including immediate and sustained pain relief.

\section{Conclusions}

RA-IVA provided better vertebral body fracture correction and reduced cement leakage than a conventional F-IVA. Although the operating time for RA-IVA was longer than for F-IVA, it decreased as the surgeon gained experience.

\section{References}

1. Barzilay Y, Schroeder JE, Hiller N, Singer G, Hasharoni A, Safran O, et al: Robot-assisted vertebral body augmentation: a radiation reduction tool. Spine (Phila Pa 1976) 39:153157,2014

2. Capel C, Fichten A, Nicot B, Lefranc M, Toussaint P, Desenclos C, et al: Should we fear cement leakage during kyphoplasty in percutaneous traumatic spine surgery? A single experience with 76 consecutive cases. Neurochirurgie 60:293-298, 2014

3. Chenin L, Capel C, Fichten A, Peltier J, Lefranc M: Evaluation of screw placement accuracy in circumferential lumbar arthrodesis using robotic assistance and intraoperative flatpanel computed tomography. World Neurosurg 105:86-94, 2017

4. Chenin L, Peltier J, Lefranc M: Minimally invasive transforaminal lumbar interbody fusion with the ROSA(TM) Spine robot and intraoperative flat-panel CT guidance. Acta Neurochir (Wien) 158:1125-1128, 2016

5. de Falco R, Bocchetti A: Balloon kyphoplasty for pure traumatic thoracolumbar fractures: retrospective analysis of 61 cases focusing on restoration of vertebral height. Eur Spine J 23 (Suppl 6):664-670, 2014

6. Delmas A, Depreux R: [Spinal curves and intervertebral foramina.] Rev Rhum Mal Osteoartic 20:25-29, 1953 (Fr)

7. Gelalis ID, Paschos NK, Pakos EE, Politis AN, Arnaoutoglou CM, Karageorgos AC, et al: Accuracy of pedicle screw placement: a systematic review of prospective in vivo studies comparing free hand, fluoroscopy guidance and navigation techniques. Eur Spine J 21:247-255, 2012 
8. Hartmann F, Gercek E, Leiner L, Rommens PM: Kyphoplasty as an alternative treatment of traumatic thoracolumbar burst fractures Magerl type A3. Injury 43:409-415, 2012

9. Hiwatashi A, Westesson PLA, Yoshiura T, Noguchi T, Togao $\mathrm{O}$, Yamashita K, et al: Kyphoplasty and vertebroplasty produce the same degree of height restoration. AJNR Am J Neuroradiol 30:669-673, 2009

10. Izadpanah K, Konrad G, Südkamp NP, Oberst M: Computer navigation in balloon kyphoplasty reduces the intraoperative radiation exposure. Spine (Phila Pa 1976) 34:1325-1329, 2009

11. Krüger A, Oberkircher L, Figiel J, Floßdorf F, Bolzinger F, Noriega DC, et al: Height restoration of osteoporotic vertebral compression fractures using different intravertebral reduction devices: a cadaveric study. Spine J 15:1092-1098, 2015

12. Lefranc M, Peltier J: Accuracy of thoracolumbar transpedicular and vertebral body percutaneous screw placement: coupling the Rosa ${ }^{\circledR}$ Spine robot with intraoperative flat-panel CT guidance - a cadaver study. J Robot Surg 9:331-338, 2015

13. Lefranc M, Peltier J: Evaluation of the ROSA ${ }^{\mathrm{TM}}$ Spine robot for minimally invasive surgical procedures. Expert Rev Med Devices 13:899-906, 2016

14. Marcus HJ, Cundy TP, Nandi D, Yang G-Z, Darzi A: Robotassisted and fluoroscopy-guided pedicle screw placement: a systematic review. Eur Spine J 23:291-297, 2014

15. McLain RF: Functional outcomes after surgery for spinal fractures: return to work and activity. Spine (Phila Pa 1976) 29:470-477, Z6, 2004

16. Mroz TE, Yamashita T, Davros WJ, Lieberman IH: Radiation exposure to the surgeon and the patient during kyphoplasty. J Spinal Disord Tech 21:96-100, 2008

17. Noriega DC, Ramajo RH, Lite IS, Toribio B, Corredera R, Ardura F, et al: Safety and clinical performance of kyphoplasty and Spine Jack ${ }^{\circledR}$ procedures in the treatment of osteoporotic vertebral compression fractures: a pilot, monocentric, investigator-initiated study. Osteoporos Int 27:2047-2055, 2016

18. Nussbaum DA, Gailloud P, Murphy K: A review of complications associated with vertebroplasty and kyphoplasty as reported to the Food and Drug Administration medical device related web site. J Vasc Interv Radiol 15:1185-1192, 2004

19. Ravi B, Zahrai A, Rampersaud R: Clinical accuracy of computer-assisted two-dimensional fluoroscopy for the percutaneous placement of lumbosacral pedicle screws. Spine (Phila Pa 1976) 36:84-91, 2011
20. Riis J, Lehman RR, Perera RA, Quinn JR, Rinehart P, Tuten $\mathrm{HR}$, et al: A retrospective comparison of intraoperative CT and fluoroscopy evaluating radiation exposure in posterior spinal fusions for scoliosis. Patient Saf Surg 11:32, 2017

21. Rotter R, Schmitt L, Gierer P, Schmitz KP, Noriega D, Mittlmeier T, et al: Minimum cement volume required in vertebral body augmentation - a biomechanical study comparing the permanent SpineJack device and balloon kyphoplasty in traumatic fracture. Clin Biomech (Bristol, Avon) 30:720-725, 2015

22. Srinivasan D, Than KD, Wang AC, La Marca F, Wang PI, Schermerhorn TC, et al: Radiation safety and spine surgery: systematic review of exposure limits and methods to minimize radiation exposure. World Neurosurg 82:1337-1343, 2014

23. Walter J, Haciyakupoglu E, Waschke A, Kalff R, Ewald C: Cement leakage as a possible complication of balloon kyphoplasty-is there a difference between osteoporotic compression fractures (AO type A1) and incomplete burst fractures (AO type A3.1)? Acta Neurochir (Wien) 154:313-319, 2012

\section{Disclosures}

Dr. Lefranc reports being a consultant for Zimmer Biomet.

\section{Author Contributions}

Conception and design: Lefranc. Acquisition of data: Lefranc, Alsalmi, Capel, Chenin. Analysis and interpretation of data: Lefranc, Alsalmi, Chenin. Drafting the article: Lefranc, Alsalmi. Critically revising the article: Lefranc, Capel. Reviewed submitted version of manuscript: all authors. Approved the final version of the manuscript on behalf of all authors: Lefranc. Statistical analysis: Lefranc. Study supervision: Lefranc, Peltier.

\section{Supplemental Information \\ Videos}

Video 1. https://vimeo.com/286006971.

\section{Correspondence}

Michel Lefranc: Amiens Picardie University Medical Center, Amiens, France. lefrancm@me.com. 